\title{
Power dependence of transient degenerate four-wave mixing in molecular systems
}

\author{
A. Pakhomov, ${ }^{1}$ Chung-Jen Wu, ${ }^{2}$ Yit-Tsong Chen, ${ }^{1,2, *}$ and S. H. Lin ${ }^{1,2}$ \\ ${ }^{1}$ Institute of Atomic and Molecular Sciences, Academia Sinica, P.O. Box 23-166, Taipei 106, Taiwan, Republic of China \\ ${ }^{2}$ Department of Chemistry, National Taiwan University, Taipei 106, Taiwan, Republic of China
}

(Received 3 September 1996)

\begin{abstract}
The object of this study is to quantitatively elucidate the laser-power dependence in transient degenerate four-wave mixing (DFWM) with an emphasis on the high laser-pump intensity region. We discuss our investigation on the power dependence of transient DFWM by taking gas-phase iodine $\left(\mathrm{I}_{2}\right)$ molecules as a testing example. The distinct physical feature is that in the high-power laser pump, where both laser-pulse duration and the inverse of pump rate are much shorter than the molecular population relaxation time, the steady-state DFWM theory of Abrams and Lind [Optical Phase Conjugation, edited by R. A. Fisher (Academic, New York, 1983), Chap. 8, pp. 211-284; Opt. Lett. 2, 94 (1978); 3, 205 (1978)] is not appropriate. The prediction by the steady-state theory has shown the DFWM to decrease with increasing laser-pump intensity as a function of $1 / I_{\text {laser }}$, which disagrees with the saturation behavior observed in the experiment. To elucidate the dependence of DFWM on the laser pump intensity, a non-steady-state extension of the nonperturbative theory of Abrams and Lind is required. The non-steady-state theoretical result will be shown to be in good agreement with the experimental power dependence at resonance transient DFWM especially in the high-power pump region. [S1050-2947(97)08703-9]
\end{abstract}

PACS number(s): 42.65.-k

\section{INTRODUCTION}

The degenerate four-wave mixing (DFWM) technique has been widely used in atomic, molecular, and solid-state spectroscopy [1-3]. It has been demonstrated by a number of authors that the implementation of various kinds of DFWM results in the striking improvement in spectral resolution and signal-to-noise ratio [4-17]. To date, the DFWM technique has been applied in many areas, such as coherent imaging, transient grating spectroscopy, and stimulated emission pumping spectroscopy. In recent years, DFWM has further emerged as a powerful optical diagnostic tool for the detection of trace species in combustion environment [4]. The technique involves the interaction of three input laser beams with a medium to produce a coherent, directional, and spectrally bright signal beam via an induced nonlinear polarization associated with the third-order susceptibility $\chi^{(3)}$ (as shown in Fig. 1).

The DFWM signal can be described as a Bragg diffraction of one of the three incoming laser beams by the grating created by the other two interfering beams in the nonlinear medium. The word "degenerate" denotes the same frequency for all of the three input laser beams and the diffracted signal radiation. In a resonant condition between the incoming laser beams and the nonlinear medium, a population grating that corresponds to a periodic spatial distribution of the excited-state population in the medium is created. The high collimation of the phase-conjugated DFWM signal permits interfering-radiation rejection, temporal and spatial sensitivity, and remote probing, which result in a unique background-free spectroscopic merit. The phase matching has also made it possible for the spectral resolution of DFWM to be sub-Doppler [18].

\footnotetext{
*Author to whom correspondence should be addressed.
}

The existing experimental and theoretical works on DFWM are so far mostly concerned with either the "steadystate" regime where the laser-pulse duration is longer than the electronic relaxation time of the medium, or the "shortpulse" case where the pulse duration is too short to create a substantial change in the population of electronic state. The theoretical treatments of DFWM can be categorized into perturbative and nonperturbative models. In the perturbative approach, the DFWM amplitude is calculated from the iterative solution of Liouville equation with a density-matrix method [5,19-22]. This approach proved to be very important in understanding the dependence of the DFWM signal on molecular electronic, vibrational, and rotational quantum numbers, the effect of atomic or molecular motion and degenerate-level coherence. It fails, however, in the case of a high-power laser pump, where the strong saturation in molecular-excited electronic states becomes essential. A nonperturbative theoretical treatment is required for this case of the high-power laser pump. The most successful nonperturbative approach is the steady-state two-level saturated absorber model derived by Abrams and Lind $[1,23,24]$. To date, the model has been shown to be in broad agreement with a large number of experimental data.

In the experiments with high-power pulsed lasers, where the pulse duration is too short to establish a steady-state condition, a nonperturbative non-steady-state theoretical approach is needed. In a previous publication [25], we applied

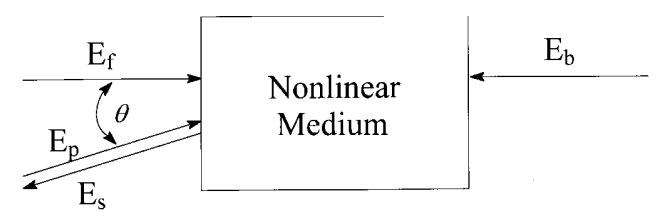

FIG. 1. Diagram of degenerate four-wave mixing. $E_{f}$ and $E_{b}$, pumps; $E_{p}$, probe; $E_{s}$, signal. 


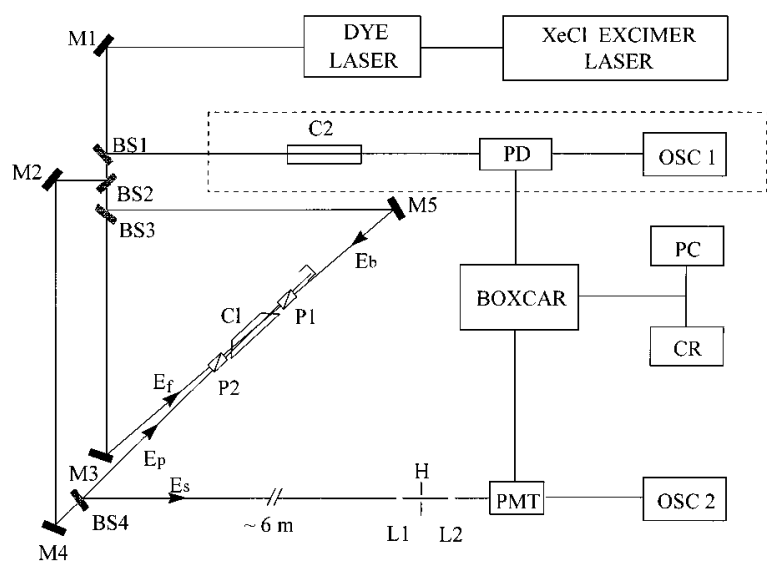

FIG. 2. Experimental setup. BOXCAR, gated integrator and boxcar averager; BS, beam splitter; $C, \mathrm{I}_{2}$ sample cell; $\mathrm{CR}$, chart recorder; $H$, pinhole; $L$, lens; $M$, mirror; OSC, oscilloscope; $P$, linear polarizer; PC, personal computer; PD, photodiode; PMT, photomultiplier tube.

a nonperturbative non-steady-state theory to treat the nonstationary evolution of the excited-state population in transient DFWM, where the optical pump rate is comparable to or faster than the excited-state decay rate. The object of this study is to elucidate quantitatively the laser-power dependence in transient DFWM with an emphasis on the high pump intensity region. We will discuss our investigation of transient DFWM by taking gas-phase iodine $\left(\mathrm{I}_{2}\right)$ molecules as a testing example. It will be shown that in high-power laser pump region, where both laser-pulse duration and the inverse of pump rate are much shorter than the molecular population relaxation time, the steady-state DFWM theory of Abrams and Lind is not adequate.

In Sec. II of this paper, experimental details are described. In the experiment, we have measured the dependence of DFWM intensities of $\mathrm{I}_{2}$ (represented as $\mathrm{I}_{\mathrm{DFWM}}$ in the following) on the interacting laser-pulse energy. A nonperturbative non-steady-state theory of DFWM derived with a densitymatrix method is briefly described in Sec. III. Comparisons between the experimental and theoretical (including the steady-state and non-steady-state) results are discussed in Sec. IV. Concluding remarks about the nonperturbative nonsteady-state theory of this work as compared to other theoretical treatments are presented in Sec. V.

\section{EXPERIMENTAL DETAILS}

The apparatus used in the present experiment for DFWM spectroscopy of $I_{2}$ is depicted in Fig. 2, and is identical to the one previously described [25]. A tunable dye laser (LambdaPhysik, Model LPD3002) with $\sim 0.04-\mathrm{cm}^{-1}$ spectral resolution, $\sim 18$-ns pulse width and $10-\mathrm{Hz}$ repetition rate, pumped by a pulsed excimer laser (Lambda-Physik, Model LPX205i), was employed in the experiment. The output of the dye laser was beam split into two pumps (labeled $E_{f}$ and $E_{b}$ in Fig. 2) and a probe $\left(E_{p}\right)$ with an energy ratio of 10:10:1 or $7: 6: 3$ in two separate measurements. The pump laser beams were counterpropagating, and the probe laser beam was aligned at a very small crossing angle $\left(\theta \sim 0.5^{\circ}\right)$ against the pump beams. The pump and probe beams were

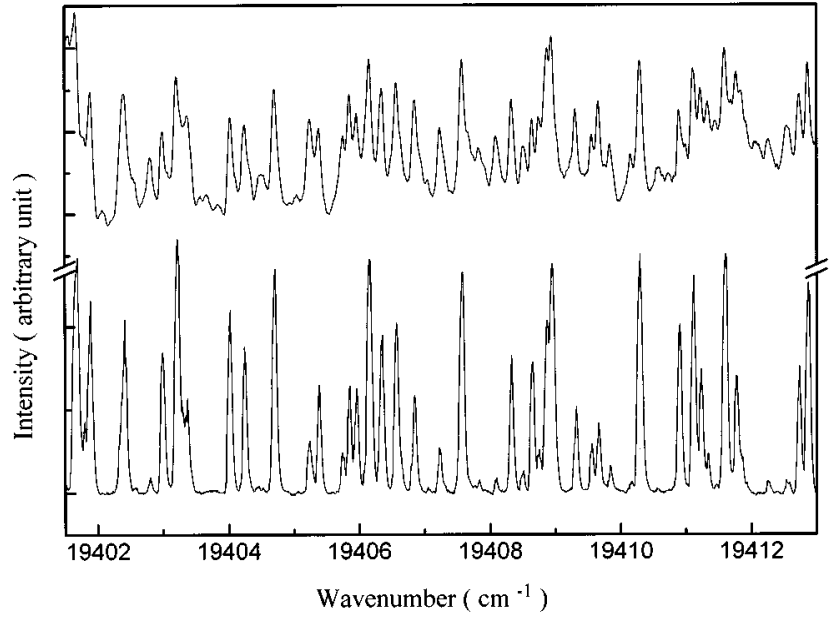

FIG. 3. Observed DFWM spectrum of $I_{2}$ with a pressure of $\sim 30 \mathrm{~m}$ Torr and a laser spectral resolution of $\sim 0.04 \mathrm{~cm}^{-1}$. The upper trace represents a direct absorption spectrum and the lower a DFWM. The average half-widths at half maximum are $\Delta \nu_{\mathrm{HWHM}} \sim 0.10 \mathrm{~cm}^{-1}$ and $\Delta \nu_{\mathrm{HWHM}} \sim 0.07 \mathrm{~cm}^{-1}$ for the upper and the lower spectra, respectively.

adjusted to be time coincident in the sample cell. The three laser beams were thus collimated to a size of $\sim 3 \mathrm{~mm}$ in diameter, and polarized parallel to each other in present experiment.

The phase-conjugated signal beam ( $E_{s}$ in Fig. 2), diffracted from the nonlinear medium, was singled out partially by a beam splitter (BS4) and traveled $\sim 6 \mathrm{~m}$ before striking a short focal lens $(f=50 \mathrm{~mm})$ that focused the beam through a pinhole ( $50 \mu \mathrm{m}$ in diameter). With spatial filtering by the pinhole, scattered light was sufficiently eliminated. The DFWM signal was finally detected by a photomultiplier tube (Hamamatsu, Model 1P28), and processed in a gated integrator and boxcar averager (Stanford, Model SR250).

In the DFWM experiment, a temperature-controlled sample cell was designed to adjust the vapor pressure of $I_{2}$. The sample cell (20 mm in diameter and $30 \mathrm{~cm}$ long), filled with solid iodine and its equilibrium vapor pressure, was covered with a water jacket, where an aqueous solution of ethyleneglycol (the volume ratio is 1:1) was circulated. The temperature of the aqueous solution was maintained at $0{ }^{\circ} \mathrm{C}$ throughout the measurements, controlled by a refrigerated circulating bath (Neslab, Model RBC-3). The corresponding $\mathrm{I}_{2}$ vapor pressure is $\sim 30 \mathrm{~m}$ Torr at $0{ }^{\circ} \mathrm{C}$. The temperature of the aqueous solution was read from a temperature probe (Keithley, Model 1301).

To calibrate the transition frequency and to select a single rovibronic transition of $\mathrm{I}_{2}$ for the measurement of power dependence in DFWM, a linear absorption spectrum of $\mathrm{I}_{2}$ was taken simultaneously in our experiment. The absorption spectrum of $\mathrm{I}_{2}$ was measured with an optical path of $\sim 30 \mathrm{~cm}$, as shown in the dashed panel in Fig. 2. The observed DFWM and linear absorption spectra of $\mathrm{I}_{2}$ are shown in Fig. 3. The wave number in the $\mathrm{I}_{2}$ spectrum was calibrated with an $\mathrm{I}_{2}$ atlas [26]. The rotational and vibrational quantum numbers for the rovibrational transitions in $\mathrm{I}_{2} \widetilde{B}^{3} \Pi_{0_{u}^{++}} \leftarrow \widetilde{X}^{1} \Sigma_{g}^{+}$were followed from the spectral assignment by Simmons and Hougen [27]. 
In the experiment, we tuned the laser in resonance with the single rovibronic transition $\left(\nu=18899.84 \mathrm{~cm}^{-1}\right)$ of $\mathrm{I}_{2} P(61) \widetilde{B}^{3} \Pi_{0_{u}^{+}}\left(v^{\prime}=34\right) \leftarrow \widetilde{X}^{1} \Sigma_{g}^{+}\left(v^{\prime \prime}=0\right)$ to determine the dependence of DFWM on the interacting laser-pulse energy. The laser energy from the pulsed dye laser was measured with a joule meter (Lambda-Physik, Model LPA 100).

\section{THEORY}

The conventional DFWM theory developed by Abrams and Lind is based on the steady-state solution of two-level density-matrix equations $[1,23,24]$. In the DFWM experiments with short pulsed lasers and long molecular relaxation time, it is desirable to take into account the non-steady-state effect. In order to compare our non-steady-state result with the steady-state prediction obtained from the Abrams-Lind model, we restricted our approach to a two-level system as well in this study. The theoretical treatment of DFWM involving the magnetic sub-levels of molecular rovibronic quantum states is described in our previous work [25]. We will demonstrate the difference between Abrams and Lind's steady-state and our non-steady-state results in the power dependence especially for the high-power laser-pump region.

We consider a system interacting with the three laser radiation fields. The Liouville equation of the system is given by

$$
\frac{\partial \hat{\rho}}{\partial t}=-i \hat{L}_{0} \hat{\rho}-i \hat{L}^{\prime}(t) \hat{\rho}-\hat{\Gamma} \hat{\rho}
$$

where $\hat{\rho}, \hat{L}_{0}, \hat{L}^{\prime}$, and $\hat{\Gamma}$ represent the density matrix, zerothorder Liouville operator, interacting Liouville operator, and damping operator, respectively [28]. Notice that (see Fig. 1) the interacting Hamiltonian is

$$
V(t)=-\sum_{i=f, b, p} \boldsymbol{\mu} \cdot \mathbf{E}_{i}(t)
$$

and

$$
\mathbf{E}_{i}(t)=\frac{1}{2}\left[\mathbf{E}_{i}(\omega) e^{i \omega t}+\text { c.c. }\right],
$$

where $\boldsymbol{\mu}$ is the dipole operator for the nonlinear medium, and $\mathbf{E}_{i}$ are the electric fields of the interacting laser beams. The subscripts $f, b$, and $p$ represent forward pump, backward pump, and probe laser beams, respectively. In the following, we will consider the case that the linear polarizations of all incoming beams are parallel to each other in accord with the present experimental condition. For a two-level model within the rotating-wave approximation, we have

$$
\begin{gathered}
\frac{\partial \Delta \rho}{\partial t}=-\frac{i}{\hbar}\left\{\left[\boldsymbol{\mu}_{12} \cdot \mathbf{E}(\omega)\right]^{*} \rho_{12}-\left[\boldsymbol{\mu}_{12} \cdot \mathbf{E}(\omega)\right] \rho_{21}\right\} \\
-\frac{\Delta \rho-\Delta \rho_{0}}{T}, \\
\frac{\partial \rho_{12}}{\partial t}=-(\Gamma+i \delta) \rho_{12}-\frac{i\left[\boldsymbol{\mu}_{12} \cdot \mathbf{E}(\omega)\right]}{2 \hbar} \Delta \rho
\end{gathered}
$$

$$
\rho_{12}=\rho_{21}^{*}
$$

We denote by $\Delta \rho=\rho_{11}-\rho_{22}$ the population difference between the ground (represented by 1) and excited (2) states of the nonlinear gas-phase medium, by $\Delta \rho_{0}=\Delta \rho(t=0)$ the equilibrium population difference, by $\delta=\omega-\omega_{21}\left(\omega_{21}\right.$ is the transition frequency) the detuning from the resonance frequency, and by $\Gamma$ and $T$ the molecular total dephasing rate and longitudinal relaxation time, respectively. Similar to that treated by Sargent, Scully, and Lamb [29], we consider laser frequency fluctuations as an additional contribution to the dephasing rate beside the collision-induced dephasing in molecules. This approach is also consistent with the phase diffusion model of Georges [30] and Anderson et al. [31] in describing broadband lasers. For simplicity of comparison, the effect of translational motion of molecules has been ignored but can easily be included [25].

As treated by Abrams and Lind [1,23,24], we regard the probing field $\mathbf{E}_{p}(\omega)$ as a perturbation, i.e., we set

$$
\mathbf{E}^{(0)}(\omega)=\mathbf{E}_{f}(\omega)+\mathbf{E}_{b}(\omega)
$$

and

$$
\mathbf{E}^{(1)}(\omega)=\mathbf{E}_{p}(\omega)
$$

The solution for the system can be significantly simplified by taking into account the characteristic values of the system. First, the laser linewidth in the present experiment is $\Delta \nu_{\text {laser }} \sim 0.04 \mathrm{~cm}^{-1}$, which is larger than the Doppler broadening of $\sim 0.01 \mathrm{~cm}^{-1}$. The molecular-velocity dependence therefore can be neglected. Second, in an application of the phase-diffusion model, the dephasing time estimated from the laser linewidth, $\tau_{d}=\left(\Gamma_{\text {laser }}\right)^{-1} \approx\left(\pi \Delta \nu_{\text {laser }}\right)^{-1} \sim 0.2 \mathrm{~ns}$, is much shorter than the laser-pulse duration of $\tau=18 \mathrm{~ns}$. Being interested in a time scale larger than $\tau_{d}$, we can replace Eq. (5) by a "coarse-grain" relation, and obtain the solution to the zeroth order of the probe field,

$$
\rho_{12}^{(0)}(\omega)=-\frac{i \boldsymbol{\mu}_{12} \mathbf{E}^{(0)}(\omega)}{2 \hbar(\Gamma+i \delta)} \Delta \rho^{(0)} .
$$

Substituting this relation into Eq. (4) yields an equation for the evolution of the population grating,

$$
\frac{\partial \Delta \rho^{(0)}}{\partial t}=-W \Delta \rho^{(0)}-\frac{\Delta \rho^{(0)}-\Delta \rho_{0}}{T},
$$

where

$$
W(\omega)=\frac{\Gamma}{\hbar^{2}\left(\Gamma^{2}+\delta^{2}\right)}\left|\boldsymbol{\mu}_{12} \cdot \mathbf{E}^{(0)}\right|^{2}
$$

is the pumping rate. Solving Eq. (10) yields

$$
\Delta \rho^{(0)}=\frac{\Delta \rho_{0}}{1+W T}\left[1+W T \exp \left\{-t\left(W+\frac{1}{T}\right)\right\}\right]
$$

Similarly, the equations to the first order of the probe field are 


$$
\begin{aligned}
\rho_{12}^{(1)}(\omega)= & -\frac{i}{2 \hbar(\Gamma+i \delta)}\left[\boldsymbol{\mu}_{12} \cdot \mathbf{E}^{(0)}(\omega) \Delta \rho^{(1)}\right. \\
& \left.+\boldsymbol{\mu}_{12} \cdot \mathbf{E}^{(1)}(\omega) \Delta \rho^{(0)}\right]
\end{aligned}
$$

and

$$
\begin{aligned}
\frac{\partial \Delta \rho^{(1)}}{\partial t}= & -\left(W+\frac{1}{T}\right) \Delta \rho^{(1)}-\frac{\Delta \rho^{(0)} \Gamma}{\hbar^{2}\left(\Gamma^{2}+\delta^{2}\right)}\left\{\left(\boldsymbol{\mu}_{12} \cdot \mathbf{E}^{(0)}\right)^{*}\right. \\
& \left.\times\left(\boldsymbol{\mu}_{12} \cdot \mathbf{E}^{(1)}\right)+\left(\boldsymbol{\mu}_{12} \cdot \mathbf{E}^{(1)}\right)^{*}\left(\boldsymbol{\mu}_{12} \cdot \mathbf{E}^{(0)}\right)\right\} .
\end{aligned}
$$

Substituting the solution of Eq. (14) into Eq. (13) and calculating the nonlinear susceptibility

$$
\mathbf{P}=\operatorname{Tr}(\boldsymbol{\mu} \hat{\rho})=\boldsymbol{\mu}_{12} \rho_{12}^{(1)}+\text { c.c. },
$$

we obtain the contribution of phase-conjugated DFWM to the polarization,

$$
\mathbf{P}_{\mathrm{pc}}(r, t)=\mathbf{K}(\omega) A_{f} A_{b} A_{p}^{*} e^{-i \omega t+i \mathbf{k}_{p} \mathbf{r}} R(t) .
$$

In Eq. (16), $A_{i}(i=f, b$, and $p)$ are the complex amplitudes of electromagnetic wave represented as

$$
\mathbf{E}_{i}=\mathbf{e} A_{i} e^{-i \mathbf{k}_{i} \mathbf{r}}
$$

with e being the unit polarization vector,

$$
\mathbf{K}(\omega)=i \boldsymbol{\mu}_{12}\left(\boldsymbol{\mu}_{12} \cdot \mathbf{e}\right)^{2}\left(\boldsymbol{\mu}_{12} \cdot \mathbf{e}\right) * \frac{\Gamma T \Delta \rho_{0}}{2 \hbar^{3}\left(\Gamma^{2}+\delta^{2}\right)(\Gamma+i \delta)},
$$

and

$$
R(t)=\frac{1-\exp \left\{-t\left(W+\frac{1}{T}\right)\right\}}{(1+W T)^{2}}+\frac{W t \exp \left\{-t\left(W+\frac{1}{T}\right)\right\}}{1+W T} .
$$

It is very important to note that we recover the steady-state result of Abrams and Lind $[1,23,24]$ by letting $t \rightarrow \infty$.

For optically thin media under the slow-varying amplitude approximation, the amplitude of the phase-conjugated DFWM signal is equal to

$$
A_{s}=\frac{2 \pi i \omega}{c} L|\mathbf{K}(\omega)| A_{f} A_{b} A_{p}^{*} R(t),
$$

where $L$ is the interaction length between the lasers and the nonlinear medium. The observed DFWM signal is to be time averaged for the square of this amplitude,

$$
S_{\mathrm{DFWM}} \sim \int_{0}^{\tau}\left|A_{s}\right|^{2} d t \sim I^{3} \int_{0}^{\tau}|R(t)|^{2} d t,
$$

where $I^{3}$ is the total input beam intensity (i.e., including the $E_{f}, E_{b}$, and $E_{p}$ beams), and $\tau$ is the laser-pulse duration. For the high-power pump experimental condition

$$
\frac{\tau}{T}\left(\frac{I_{\text {pump }}}{I_{\text {sat }}}\right)^{2} \gg 1
$$

where

$$
I_{\text {pump }}=\frac{c}{8 \pi}\left|\mathbf{E}^{(0)}\right|^{2}
$$

is the pump intensity and

$$
I_{\mathrm{sat}}=\frac{c \hbar^{2} \Gamma}{8 \pi \mu_{12}^{2} T}
$$

is the saturation intensity, the contribution from the first term (steady state) of Eq. (19) to the integral in Eq. (21) is small comparing with that from the second one ("spike") of Eq. (19). The resulting expression for the DFWM signal can thus be reduced to

$$
S_{\mathrm{DFWM}} \approx I^{3} \int_{0}^{\tau} t^{2} \exp \left\{-\frac{2 I_{\mathrm{pump}} t}{I_{\mathrm{sat}} T}\right\} d t \propto \int_{0}^{\eta / \eta_{0}} x^{2} e^{-2 x} d x,
$$

where $\eta=I_{\text {pump }} \tau$ is the total pump pulse energy per unit area, and

$$
\eta_{0}=I_{\mathrm{sat}} T=\frac{c \hbar^{2} \Gamma}{8 \pi \mu_{12}^{2}}
$$

\section{RESULTS AND DISCUSSION}

The experimental measurements for the dependence of the DFWM signal intensity $\left(\mathrm{I}_{\mathrm{DFWM}}\right)$ on interacting laserpulse energy are plotted in Fig. 4, where the abscissa represents the laser-pulse energy of the forward pump beam. The energy ratios for the forward, backward, and probe beams are 10:10:1 and 7:6:3, respectively, in Figs. 4(a) and 4(b). As shown in the figures, $\mathrm{I}_{\mathrm{DFWM}}$ becomes saturated as the laser energy increases. When the forward pulse energy is beyond $\sim 100 \mu \mathrm{J}$, the observed DFWM does not grow pronouncedly any longer.

To explain the saturation of DFWM in the present experiment qualitatively, we note that the last integral in Eq. (25) is convergent and monotonically reaches an upper limit, which reveals the saturation behavior of $\mathrm{I}_{\mathrm{DFWM}}$ in the high-power laser-pump region. For a quantitative comparison between the non-steady-state theoretical and experimental results, we need to perform some numerical calculations. The dephasing rate, according to the laser linewidth of $\Delta \nu_{\text {laser }}$ $\sim 0.04 \mathrm{~cm}^{-1}$, corresponds to $\Gamma \approx \pi \Delta \nu_{\text {laser }} \sim 4 \times 10^{9} \mathrm{~s}^{-1}$. The collisional dephasing rate of the $I_{2}$ molecules is about $10^{6} \mathrm{~s}^{-1}$ estimated from Ref. [32] for the present experimental condition. The longitudinal relaxation time of $I_{2} \widetilde{B}^{3} \Pi_{0_{u}^{+}}, \sim 1 \mu \mathrm{s}$, is much longer than the laser-pulse duration of $18 \mathrm{~ns}$. The rovibronic transition moment was calculated from the relation

$$
\mu^{2}=\frac{1}{3} \frac{J^{\prime \prime}}{2 J^{\prime \prime}+1}\left|\left\langle v^{\prime \prime} \mid v^{\prime}\right\rangle\right|^{2} \mu_{e}^{2},
$$

where $J^{\prime \prime}$ is the rotational quantum number of the lower state in the transition, $\left|\left\langle v^{\prime \prime} \mid v^{\prime}\right\rangle\right|^{2}$ is the Franck-Condon factor, and $\mu_{e}$ is the electronic transition dipole moment. Using the experimental data of Refs. [32-34], we estimated the square of electronic matrix element $\mu_{e}^{2} \approx 0.9 \mathrm{D}^{2}$ and the Franck-Condon factor $\left|\left\langle v^{\prime \prime}=0 \mid v^{\prime}=34\right\rangle\right|^{2} \approx 0.03$ for 

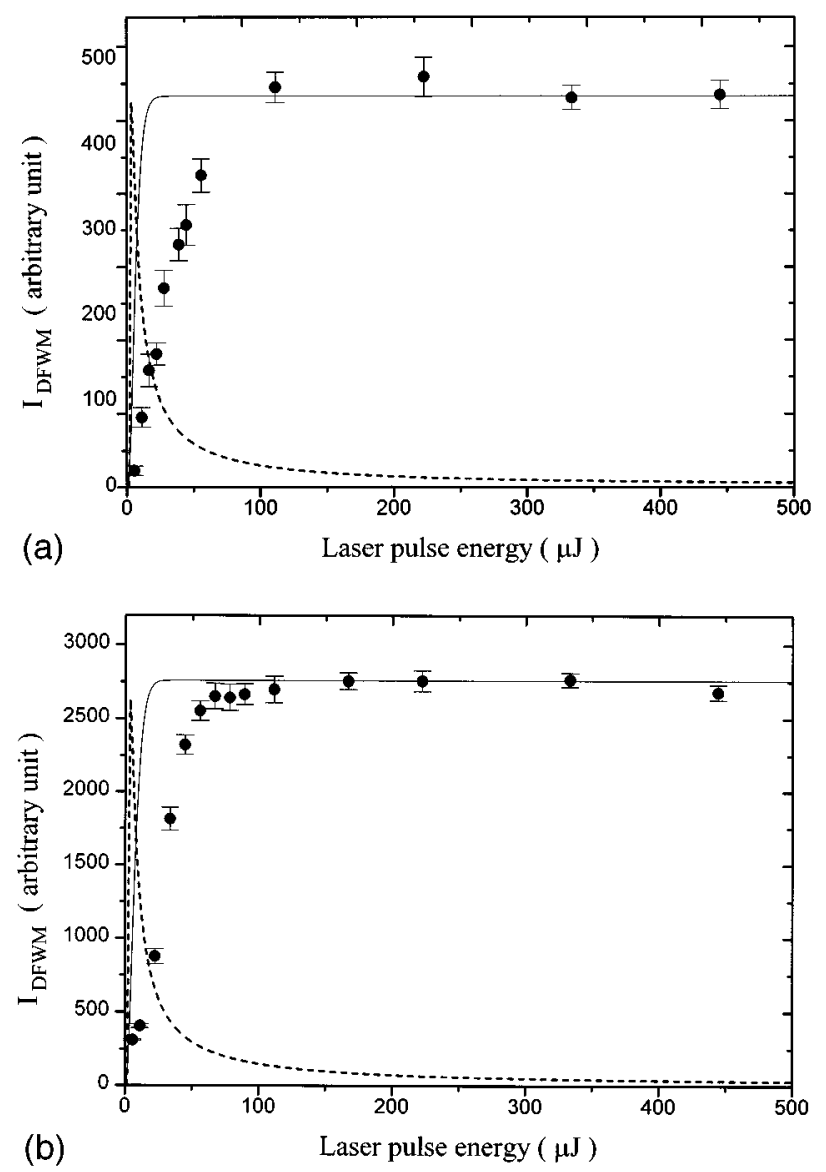

FIG. 4. Dependence of the DFWM signal on the interacting laser-pulse energy: $\bigcirc$, experimental data; - , theoretical result from this work; and -- -, theoretical result of Abrams and Lind. The vapor pressure of $\mathrm{I}_{2}$ molecules was maintained at $\sim 30 \mathrm{~m}$ Torr throughout the measurements. The experiments were carried out with a laser spectral resolution of $\sim 0.04 \mathrm{~cm}^{-1}$. The laser was tuned in resonance with the single rovibronic transition $\left(\nu=18899.84 \mathrm{~cm}^{-1}\right)$ of $\mathrm{I}_{2} P(61) \widetilde{B}^{3} \Pi_{0_{u}^{+}}\left(v^{\prime}=34\right)$ $\leftarrow \widetilde{X}^{1} \Sigma_{g}^{+}\left(v^{\prime \prime}=0\right)$. The abscissa represents the energy of the forward pump beam $\left(E_{f}\right)$. The energy ratio for the forward pump, backward pump, and probe laser beams has been maintained as (a) 10:10:1 and (b) 7:6:3, respectively, in the two separate measurements.

$I_{2} \widetilde{B}^{3} \Pi_{0_{u}^{+}}\left(v^{\prime}=34\right) \leftarrow \widetilde{X}^{1} \Sigma_{g}^{+}\left(v^{\prime \prime}=0\right)$. For the rovibronic transition moment of $\mathrm{I}_{2} P(61) \widetilde{B}^{3} \Pi_{0_{u}^{+}}\left(v^{\prime}=34\right)$ $\leftarrow \widetilde{X}^{1} \Sigma_{g}^{+}\left(v^{\prime \prime}=0\right)$, we obtain $\mu^{2} \approx 0.004 \mathrm{D}^{2}$.

In the calculation, we computed the power dependence of the DFWM signal intensity using the above parameters and Eqs. (16)-(26). The comparison between the non-steadystate theoretical (solid line) and experimental (solid circle) results is shown in Fig. 4. The agreement between the theoretical and experimental values can be regarded as fairly good, noting especially that we have assumed the $100 \%$ overlap of all incoming laser beams in the calculation. A correction to this assumption should lead to the decrement of the effective pump rate, and therefore to slower rising of the theoretical curve in the low laser-pump energy region. Other corrections, such as the effect of grating washout by molecular motion and the involvement of the magnetic sublevels in addition to the simple two-level model, could also improve the deviation in the low-power pump region between the non-steady-state theory and experimental results.

The theoretical DFWM curves (dashed line) predicted from the Abrams-Lind model are also presented in Fig. 4 for comparison. The inadequacy of the Abrams-Lind theory for the present experimental DFWM conditions is apparently seen. In low-power pump region, the Abrams-Lind's result is close to the cubic dependence of laser energy predicted from the perturbative theory. In the high-power pump region, both perturbative theory and Abrams-Lind model deviate seriously from the experimental measurements.

\section{CONCLUDING REMARKS}

The basic feature of transient DFWM considered in this paper is that the power dependence of DFWM gains saturation under high-power laser pump as depicted in Fig. 4. This saturation phenomenon is essentially different from the theoretical predictions of the steady-state theory by Abrams and Lind $[1,23,24]$, where $\mathrm{I}_{\mathrm{DFWM}}$ decreases with increasing laser pump intensity $\mathrm{I}_{\text {laser }}$ as a function of $1 / \mathrm{I}_{\text {laser }}$. The inadequacy of the Abrams and Lind model is due to the fact when the optical pump rate is comparable to or faster than the molecular excited-state decay rate, the steady-state treatment is no longer appropriate. As demonstrated in this paper, a nonsteady-state effect should be introduced to deal with the nonstationary evolution of the excited-state population in transient DFWM. In this work, we showed that the theoretical power dependence of DFWM, i.e., the integral in Eq. (25), reaches an upper limit in the high-power laser-pump region. Mathematically, the steady-state outcome obtained by Abrams and Lind is resulted from setting $\partial \Delta \rho / \partial t$ in Eq. (4) of this paper equal to zero. This has been proved in Eqs. (16)-(19), where the result of steady-state theory can be recovered from our non-steady-state formula by letting $t \rightarrow \infty$.

The saturation in DFWM, resulting from the saturation of transient population in the molecular excited state, has been emphasized by a number of authors [35-37]. However, a phenomenological approach has been used in those papers, where the nonlinear polarization was taken as being simply proportional to the transient population

$$
P(t)=P_{0} \exp \left\{-\int_{-\infty}^{t} W\left(t^{\prime}\right) d t^{\prime}\right\} .
$$

As compared with our result, this phenomenological approach cannot explain the saturation behavior of DFWM in the high-power pump at the present experimental condition. Another approach studied by Meacher et al. [38] with steady-state statistical theory has also shown the decrease of $\mathrm{I}_{\text {DFWM }}$ with increasing laser pump intensity. In the laserpulse energy scale of $W T \sim 10^{2}$, which is comparable to our experimental condition, Meacher et al. predicted the decrease of DFWM as a function of $1 / \mathrm{I}_{\text {laser }}^{2}$ The steady-state statistical theory also disagrees with the experimental result.

A recent paper by Syed, Crofts, and Damzen [39] for the study of DFWM in saturable Ti:sapphire gain media with finite probe is worth noting. In their paper, they reported a fall-off DFWM efficiency with an increasing pump energy, which is different from our observation of the saturation of 
DFWM in $\mathrm{I}_{2}$ molecules. The DFWM experimental condition reported in Ref. [39] is for saturable media with finite probe beam, whereas the probe intensity in our experiment increases with the increasing pump energy at a fixed proportion. The fall-off of DFWM efficiency in the study of Ref. [39] can actually be qualitatively explained by our theory with slight modification. By multiplying a factor of $I_{\text {probe }} / I_{\text {pump }}$ in Eq. (25) of this paper, where $I_{\text {probe }}$ is constant, the fall-off of the DFWM power-dependence curve with increasing pump energy is apparently seen.

Finally, we would like to point out that the non-steadystate DFWM theory considered in the present paper is valid in the case where both laser-pulse duration and the inverse of the pump rate $(1 / W)$ are much shorter than the molecular relaxation time. To fulfill the fast pump and slow relaxation condition, the non-steady-state DFWM theory is more suitable for gas-phase systems than the condensed phase, where fast relaxations usually take place. In an opposite extreme, for instance at very high gas pressure or in condensed matter, the steady-state term of Eq. (19) becomes dominant and the power dependence of DFWM can be described by the Abrams-Lind model in a satisfying way. Although in this paper we focus our attention on the power dependence of DFWM, the non-steady-state theory of DFWM can be used to study the pressure dependence, polarization effect and band shape of DFWM.

\section{ACKNOWLEDGMENTS}

We wish to thank Shwu-Chyi Lin and Chung-Hung Chang for their experimental contributions in the early study. This work was supported, in part, by the National Science Council of Republic of China under Grant Nos. NSC-862113-M-001-030 and NSC-86-2113-M-001-043 CT.
[1] Optical Phase Conjugation, edited by R. A. Fisher (Academic, New York, 1983), Chap. 8, pp. 211-284.

[2] H. J. Eichler, P. Gunter, and D. W. Pohl, Laser-Induced Dynamic Gratings (Springer, Berlin, 1986).

[3] J. F. Reitjes, Nonlinear Optical Parametric Processes in Liquids and Gases (Academic, New York, 1984).

[4] For the references of DFWM, see the review article by G. Hall and B. J. Whitaker, J. Chem. Soc. Faraday Trans. 90, 1 (1994), and some more recent publications (only to our knowledge), such as the following Refs. [5-17].

[5] S. Williams, R. N. Zare, and L. A. Rahn, J. Chem. Phys. 101, 1072 (1994).

[6] S. Williams, R. N. Zare, and L. A. Rahn, J. Chem. Phys. 101, 1093 (1994).

[7] J. Ishii, T. Matsui, K. Tskiyama, and K. Uehara, Chem. Phys. Lett. 220, 29 (1994).

[8] P. DeRose, H. L. Dai, and P. Y. Cheng, Chem. Phys. Lett 220, 207 (1994).

[9] B. Ai, D. S. Glassner, and R. J. Knize, Phys. Rev. A 50, 3345 (1994).

[10] M. A. Buntine, D. W. Chandler, and C. C. Hayden, J. Chem. Phys. 102, 2718 (1995).

[11] M. N. R. Ashfold, D. W. Chandler, C. C. Hayden, R. I. McKay, and A. J. R. Heck, Chem. Phys. 201, 237 (1995).

[12] H. Failache, M. Oriá, and A. Lezama, Phys. Rev. A 52, 699 (1995).

[13] L. Lehr, M. Motzkus, G. Pichler, K. L. Kompa, and P. Hering, J. Chem. Phys. 104, 9698 (1996).

[14] Y. Tang and S. A. Reid, Chem. Phys. Lett. 248, 476 (1996).

[15] R. M. Helm, R. Neuhauser, and H. J. Neusser, Chem. Phys. Lett. 249, 365 (1996).

[16] B. Hemmerling, R. Bombach, and W. Hubschmid, Chem. Phys. Lett. 256, 71 (1996).

[17] E. Pawelec, W. Gawlik, B.Samson, and K. Musiol, Phys. Rev. A 54, 913 (1996).

[18] M. Ducloy and D. Bloch, Phys. Rev. A 30, 3107 (1984).

[19] J. F. Lam and R. L. Abrams, Phys. Rev. A 26, 1539 (1982).
[20] A. I. Alekseev, Zh. Eksp. Teor. Fiz. 101, 435 (1992) [Sov. Phys. JETP 74, 227 (1992)].

[21] P. R. Berman, D. G. Steel, G. Khitrova, and J. Liu, Phys. Rev. A 38, 252 (1988).

[22] P. Fu, Z. Yu, X. Mi, and P. Ye, J. Phys. (Paris) 48, 2089 (1987).

[23] R. L. Abrams and R. C. Lind, Opt. Lett. 2, 94 (1978).

[24] R. L. Abrams and R. C. Lind, Opt. Lett. 3, 205 (1978).

[25] A. Pakhomov, C.-J. Wu, Y.-T. Chen, and S. H. Lin, Opt. Quantum Electron 28, 1477 (1996).

[26] S. Gerstenkorn and P. Luc, Atlas du Spectre d'Absorption de la Molecule de l'Iode entre $17500-20000 \mathrm{~cm}^{-1}$ (CRNS, Paris, 1978).

[27] J. D. Simmons and J. T. Hougen, J. Res. Nat. Bur. Stand. Sec. A 81, 25 (1977).

[28] Y. R. Shen, The Principle of Nonlinear Optics (Wiley, New York, 1984).

[29] M. Sargent III, M. O. Scully, and W. E. Lamb, Jr., Laser Physics (Addison-Wesley, London, 1974), pp. 84-89 and 301-340.

[30] A. T. Georges, Phys. Rev. A 21, 2034 (1980).

[31] M. H. Anderson, R. D. Jones, J. Cooper, S. J. Smith, D. S. Elliott, H. Ritsch, and P. Zoller, Phys. Rev. A 42, 6690 (1990).

[32] C. Colón, M. Ortiz, and J. Campos, J. Mol. Spectrosc. 112, 357 (1985).

[33] A. Chutjian and T. C. James, J. Chem. Phys. 51, 1242 (1969).

[34] F. Martin, R. Bacis, S. Churassy, and G. Verges, J. Mol. Spectrosc. 116, 71 (1986).

[35] J. G. Diels, I. McMichael, and H. Vanherzeele, IEEE J. Quantum Electron. QE-20, 630 (1984).

[36] A. Brignon and J.-P. Huignard, Opt. Commun. 110, 717 (1994).

[37] K. S. Syed, R. P. M. Green, G. J. Crofts, and M. J. Domzen, Opt. Commun 112, 175 (1994).

[38] D. R. Meacher, A. Charlton, P. Ewart, J. Cooper, and G. Alber, Phys. Rev. A 42, 3018 (1990).

[39] K. S. Syed, G. J. Crofts, and M. J. Damzen, J. Opt. Soc. Am. B 13, 1892 (1996). 